EDITOR'S

CHOICE

\title{
Midportion Achilles tendinosis and the plantaris tendon
}

\author{
Håkan Alfredson
}

\section{Correspondence to} Håkan Alfredson, Professor, Sports Medicine Unit, Department of Surgical and Perioperative Science Sports Medicine, University of Umeå, S-901 87 Umeå, Sweden; hakan.alfredson@idrott. umu.se

Accepted 13 May 2011 Published Online First 31 May 2011

\begin{abstract}
Objectives When re-operating patients with midportion Achilles tendinosis, having had a poor effect of ultrasound (US) and Doppler-guided scraping, the author found the involvement of the plantaris tendon to be a likely reason for the poor result. The aim of this study was to investigate the occurrence of a plantaris tendon in close relation to the Achilles tendon in consecutive patients with midportion Achilles tendinosis undergoing treatment with US and Doppler-guided scraping.

Material and methods This study includes 73 consecutive tendons with chronic painful midportion Achilles tendinosis, where US+Doppler examination showed thickening, irregular tendon structure, hypoechoic regions, and localised high blood flow outside and inside the ventral Achilles midportion. The tendons were treated with US+Doppler-guided scraping, via a medial incision. If there was a plantaris tendon located in close relation to the medial Achilles, it was extirpated.
\end{abstract}

Results An invaginated, or 'close by located', enlarged plantaris tendon was found in 58 of $73(80 \%)$ tendons. Preliminary clinical results of the combined procedure, US + Doppler-guided surgical scraping and extirpation of the plantaris tendon, are very promising.

Conclusions A thickened plantaris tendon located in close relation to the medial Achilles seems common in patients with chronic painful midportion tendinosis. The role of the plantaris tendon in midportion Achilles tendinosis needs to be further evaluated and should be kept in mind when treating this condition.

\section{INTRODUCTION}

During the last decade, new treatment methods for midportion Achilles tendinopathy/tendinosis have evolved. Mini-invasive surgical treatment targeting the soft tissues on the ventral side of the tendon has shown good clinical results, with possibilities for a quick return to Achilles tendon loading sports. ${ }^{12}$ At our clinic, a few patients having had a poor result of ultrasound (US) +Doppler-guided scraping were found to suffer from mainly medial midportion pain, and were found to have a thickened plantaris tendon located in close relation to the medial Achilles midportion during re-operation. After extirpation of the plantaris tendon, together again with a local scraping of the ventral Achilles, there were good clinical results.

A recent observational anatomical study showed a plantaris tendon in all 107 dissected lower extremities, and there was a close 'retinaculum-like' relationship between the Achilles midportion and the plantaris in 11 of them. ${ }^{3}$
This study aimed to investigate the occurrence of a plantaris tendon in close relation to the thickened Achilles midportion in consecutive patients with chronic painful midportion Achilles tendinosis, undergoing treatment with US and Doppler-guided scraping.

\section{MATERIAL AND METHODS}

Fifty-six consecutive patients (36 males and 20 females, mean age 43 and 52 years, respectively) with a long duration of midportion Achilles tendinopathy/tendinosis in altogether 73 tendons were included. There were 9 professional male (mean age 32 years) athletes (Football, rugby, track and field, cricket) and 4 high-level male (mean age 38 years) recreational athletes (running, skiing, track and field) with midportion tendinopathy/tendinosis in altogether 18 tendons.

\section{US and Doppler examination}

All tendons were examined with high-resolution grey-scale US and colour Doppler (CD), Acuson Sequoia (Siemens) at inclusion. A linear multifrequency (8-13 MHz) probe was used. CD was used to diagnose regions with high blood flow, and to locate where the blood flow entered the tendon. Both the Achilles tendons were examined.

\section{Surgical treatment - US and Doppler-guided scraping +extirpation of the plantaris tendon} After washing, local anaesthesia $(5-10 \mathrm{ml}$ xylocaine+adrenaline, $5 \mathrm{mg} / \mathrm{ml}$ ) was injected on the medial and ventral side of the Achilles midportion. The skin was then draped with a sterile paper-cover exposing only the midportion of the Achilles tendon.

A longitudinal skin incision $(1-2 \mathrm{~cm})$ was placed on the medial side of the Achilles midportion, and the tendon was carefully identified. If a plantaris tendon was found (figure 1), invaginated into the medial Achilles or located close by the medial or ventromedial side, it was followed and released distally and proximally, cut in both ends, and extirpated. There was often a richly vascularised fat tissue interpositioned between the Achilles and the plantaris tendon (figure 2). After removing the plantaris tendon, the traditional scraping procedure was performed. ${ }^{2}$ In the regions with US and Doppler verified changes and high blood flow, the tendon was completely released from the ventral soft tissue, by sharp dissection with a knife, staying close to the ventral tendon. This was followed by haemostasis, using diatermia. The skin was closed by a single non-resorbable sutures. 


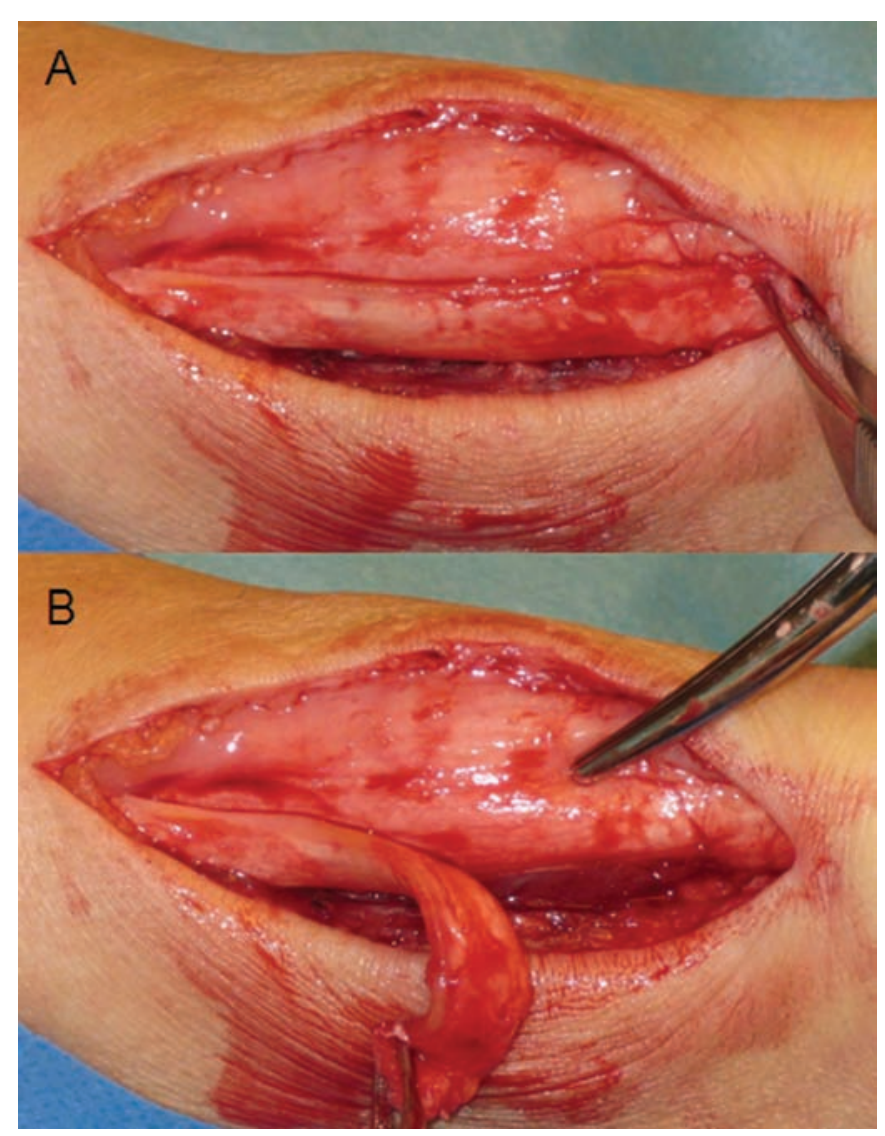

Figure 1 (A) Thickened plantaris tendon found in close relation ('invaginated') to the medial Achilles midportion in a patient with chronic painful midportion Achilles tendinosis. (B) The plantaris tendon carefully being removed from the medial Achilles. Note signs of 'invagination'.

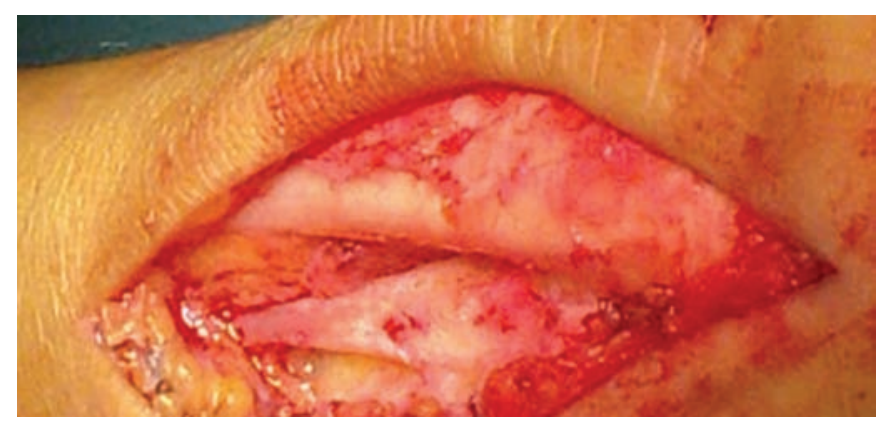

Figure 2 Thickened and widened plantaris tendon found ventral (deep side) to the medial side of the Achilles mid- and distal portion. Notice fat tissue interpositioned between the Achilles and plantaris tendons.

\section{RESULTS}

During treatment with US+Doppler-guided scraping, an invaginated, or close by located, enlarged plantaris tendon was found in 58 of the 73 tendons.

Six tendons were re-operated. In four tendons, the initial scraping operation was done via lateral incision, where the plantaris was not seen. In four tendons, the plantaris tendon was tightly coalesced with the Achilles tendon, and could not be identified at the first operation.

There are yet no longer-term clinical and sonographic followups, but preliminary clinical results of the combined procedure,

\section{What is already known on this topic}

Chronic painful midportion Achilles tendinosis can under local anaesthesia be treated successfully with the minor surgical procedure - ultrasound and Doppler-guided scraping. The plantaris tendon seems to exist in all individuals, but the position of the plantaris in relation to the medial Achilles is varying.

\section{What this study adds}

In patients with chronic painful midportion Achilles tendinosis, the plantaris tendon was found in close relation to the medial Achilles midportion in $80 \%$ of the tendons. The plantaris tendon might be of interest for the aetiology and treatment of midportion tendinosis.

US + Doppler-guided surgical scraping and extirpation of the plantaris tendon, are very promising.

\section{DISCUSSION}

To the best of our knowledge, this is the first larger material study where the plantaris tendon has been identified in close relation to the medial Achilles tendon in patients suffering from chronic painful midportion Achilles tendinosis.

The background of the findings is a recent study where patients with midportion Achilles tendinosis were treated with US and Doppler-guided scraping and the failures during re-operation were found to have a thickened plantaris tendon in close relation to the medial Achilles tendon midportion. ${ }^{2}$ In that study, the patients were operated via a lateral incision, a likely reason why the plantaris tendon involvement in these particular tendons was not detected at the first operation.

The potential role for the plantaris tendon in midportion Achilles tendinosis has not been clarified. The findings are interesting, as it recently has been shown that the plantaris is a stiffer and stronger tendon than the Achilles. ${ }^{4}$ Thereby, in cases where the plantaris is located close to the Achilles, theoretically, there can be an interference between the plantaris and the Achilles. Whether one tendon provokes the other to start the process of tendinosis is not known, but the "compression theory' 5 that is being discussed as a possible inducer for insertional tendinopathy/tendinosis might be actual also for midportion tendinopathy/tendinosis.

How important then is the plantaris tendon for symptomatic midportion Achilles tendinosis? Well, because the scraping operation alone, without extirpation of the plantaris tendon, in previous studies has shown good clinical results with very few failures, ${ }^{12}$ most likely the plantaris tendon is to be considered as a co-factor. Future randomised studies will have to investigate whether extirpation of the plantaris tendon alone is enough for good clinical results. Anyhow, it seems indicated to use a medial approach when operating, and if the plantaris tendon is found to be located in close relation to the thickened Achilles midportion, it seems appropriate to extirpate the plantaris together with the scraping procedure. We have not experienced any complications from extirpation of the plantaris together with the scraping procedure. 


\section{Competing interests None.}

Ethics approval Ethical approval was obtained from the Ethics Committee University of Umeå, Sweden.

Provenance and peer review Not commissioned; externally peer reviewed.

\section{REFERENCES}

1. Alfredson H, Ohberg L, Zeisig E, et al. Treatment of midportion Achilles tendinosis: similar clinical results with US and CD-guided surgery outside the tendon and sclerosing polidocanol injections. Knee Surg Sports Traumatol Arthrosc 2007;15:1504-9.
2. Alfredson H. Ultrasound and Doppler-guided mini-surgery to treat midportion Achilles tendinosis: results of a large material and a randomised study comparing two scraping techniques. Br J Sports Med 2011;45:407-10.

3. van Sterkenburg MN, Kerkhoffs GM, Kleipool RP, et al. The plantaris tendon and a potential role in mid-portion Achilles tendinopathy: an observational anatomical study. J Anat 2011;218:336-41.

4. Lintz F, Higgs A, Millett $M$, et al. The role of plantaris longus in Achilles tendinopathy: a biomechanical study. Foot Ankle Surg 2010;(In Press).

5. Cook JL, Purdam CR. Is tendon pathology a continuum? A pathology model to explain the clinical presentation of load-induced tendinopathy. Br J Sports Med 2009;43:409-16.

W 